\title{
Equivalence of Disturbance Observer Structures for Linear Systems
}

\author{
Erwin Schrijver ${ }^{\dagger}$, Johannes van Dijk $^{\dagger}$ and Henk Nijmeijer ${ }^{\ddagger}$ \\ †University of Twente, Department of Mechanical Engineering and Automation \\ $\ddagger$ Technical University of Eindhoven, Department of Mechanical Engineering, Systems \& Control \\ The Netherlands \\ e-mail: E.Schrijver@wb.utwente.nl / J.vanDijk@wb.utwente.nl
}

\begin{abstract}
Several techniques exists to incorporate disturbance rejection requirements in a linear controller design. Contrary to, for example the $\mathrm{H}$-infinity controller design technique where only one degree of freedom is available to obtain both disturbance rejection and performance, a Disturbance Observer adds a degree of freedom, thereby enabling a separate design of the disturbance rejection and the performance.

There are many ways to design, implement and represent disturbance observers. In this paper, we focus on two design methodologies and their corresponding representations. It can be shown that, in case the (SISO) plant is linear, the methodologies result in an equivalent disturbance observer. In this paper, we will use this equivalence to relate some properties well-known for one methodology to the other methodology, and vice versa.
\end{abstract}

\section{Introduction}

The two representations we focus on in this paper, are given in figure 1 (Umeno and Hori, 1991; Bickel and Tomizuka, 1999) and figure 2 (Johnson, 1971; Profeta et al., 1990).

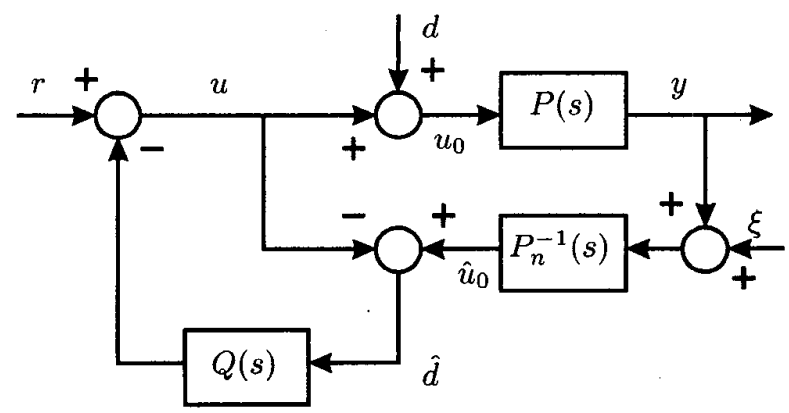

Figure 1: Disturbance Estimating Filter (DEF).
The disturbance observer in figure 1 will be referred to as a Disturbance Estimating Filter (DEF). Because of its simple structure and its logical interpretation, this disturbance observer structure is appealing for linear SISO systems. The DEF is based on using the inverse nominal plant model $P_{n}^{-1}(s)$ to estimate $u_{0}$. By feeding back the difference between $\hat{u}_{0}$ and $u$, which is thus an estimate of the disturbance $d$, disturbance rejection is accomplished. However, direct feedback of $\hat{d}$ can not be realized, as in general the inverse plant model is nonproper, and direct feedback would result in an algebraic loop. Therefore, the filter $Q(s)$ is introduced. The design of the DEF is now reduced to the design of the filter $Q(s)$.

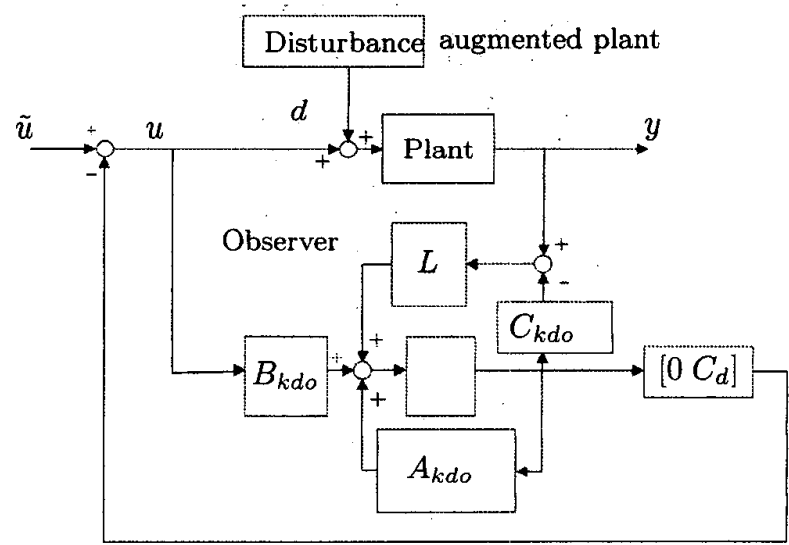

Figure 2: Kalman Disturbance Observer (KDO).

The disturbance observer structure of Figure 2, referred to as a Kalman Disturbance Observer (KDO), uses a different approach. First of all, it is assumed that the disturbance signal $d(t)$ can be thought of as being generated by a fictitious autonomous dynamic system. This fictitious disturbance generator can be included in the plant, resulting in the augmented plant. Under some mild conditions (Coelingh, 2000), an observer can be constructed which then estimates not only the states of the plant, but also the states of the 
fictitious disturbance generator. Using this estimated disturbance state, an estimate of the disturbance can be constructed and used for feedback.

In (Schrijver et al., 2000), it is shown that the DEF and the KDO are equivalent in the sense that they represent the same disturbance observer. Although exact equivalence is of no importance from an engineering point of view, it proves beneficial in that some properties wellknown for the KDO can be applied on the DEF design and vise versa.

\section{Consequences of the equivalence}

\subsection{Relative degree}

Contrary to the DEF design method, where the relative degree $\rho$ of the filter $Q(s)$ can be chosen freely (that is, under the constraint $\rho \geq \rho_{p}$ with $\rho_{p}$ the relative degree of the plant model), the KDO always implements a filter $Q(s)$ with relative degree $\rho=\rho_{p}+1$. So the KDO always implements at least $20 \mathrm{~dB} /$ decade additional high-frequency roll-off, which might be beneficial for robust stability (Schrijver and Dijk, 2000). However, it might as well be superfluous, resulting in a higher order controller then needed. The fixed relative degree also means that a DEF can not (in general) be rewritten in a $\mathrm{KDO}$ structure.

\subsection{Sensitivity functions}

An important aspect involved in Disturbance Observer designs (at least for the DEF), is the (complementary) sensitivity function. For the DEF design, it is well known that $(1-Q)$ and $Q$ represent the sensitivity and complementary sensitivity functions, respectively. Using this property of the DEF, the filter $Q(s)$ can be carefully chosen to guarantee robust stability and performance. However, little literature is available that discusses robustness for the KDO design method (see (Coelingh, 2000) for one of the exceptions). But because the KDO can be written in terms of a DEF structure, small-gain criteria can be applied for the KDO design as well such that robustness is attained.

\subsection{Pole-zero cancellation}

One of the requirements for the KDO method to succeed, is that the augmented plant model (i.e. plant and disturbance generator) is observable. To the best of our knowledge, similar requirements on pole-zero cancellation have never been reported for a DEF design. But because of the equivalence, this requirement can directly be translated to the DEF design.

\subsection{Non-minimum-phase plants}

Non-minimum-phase systems used to be problematic for a DEF design, as the zeros in the right-half-plane re-appear as right-half-plane poles in the feedback loop of a DEF. In contrast, the KDO handles non-minimumphase systems easily. It is clear that the DEF can handle non-minimum-phase systems equivalently, by virtue of the equivalence, by proper selection of the filter $Q(s)$.

\subsection{Alternative disturbance models}

When the disturbance to be rejected is not described by $1 / s^{k}$, but more generally described by $1 /\left(s^{k}+\right.$ $\left.q_{k-1} s^{k-1}+\cdots q_{0}\right)$, the KDO design method can simply be used to arrive at a disturbance observer. On the other hand, the DEF design method is less apparent. In fact, the filter $Q(s)$ has to be selected according to the very general structure:

$$
Q(s)=\frac{1+\sum g_{m} s^{m}}{1+\sum f_{m} s^{m}}
$$

such that $1 /(1-Q)$ includes the factor $1 /\left(s^{k}+\right.$ $\left.q_{k-1} s^{k-1}+\cdots q_{0}\right)$. It is not trivial to select $Q(s)$ such that this requirement, in conjunction with the common requirements imposed by the $\mathrm{DEF}$ design, are satisfied. Though, as a result of the equivalence between the DEF and the KDO, it is always possible to construct a $Q(s)$ that fulfills this requirement.

\section{References}

R. Bickel and M. Tomizuka, 1999. Passivity-Based Versus Disturbance Observer Based Robot Control: Equivalence and Stability. ASME J. of Dynamic Systems, Measurements and Control, 121 (1999), 41-47.

H.J. Coelingh, 2000. Design Support for Motion Control Systems; a Mechatronic Approach. PhD dissertation, University of Twente, The Netherlands.

C.D. Johnson, 1971. Accomodation of External Disturbances in Linear Regulator and Servomechanism Problems. IEEE Trans. Automatic Control, 16 (1971), 635644.

J.A. Profeta, W.G. Vogt, and M.H. Mickle, 1990. Disturbance Estimation and Compensation in Linear Systems. IEEE Transactions on Aerospace \& Electronic Systems, 26 (1990), 225-231.

E. Schrijver and J. Van Dijk, 2000. On the Design of Robust Disturbance Observers for Mechatronic Systems. 1st IFAC-Conference Mechatronics 2000, Darmstadt, Germany, (2000).

E. Schrijver, J. Van Dijk, and H. Nijmeijer, 2000. Equivalence of Disturbance Observer Structures for Linear Systems. Internal Report WA-724, Mechanical Engineering and Automation, University Of Twente, (2000).

T. Umeno and Y. Hori, 1991. Robust Speed Control of DC Servomotors Using Modern Two Degrees-ofFreedom Controller Design. IEEE Trans, on Industrial Electronics, 38 (1991), 363-368. 